Puhr, Kirsten

Drei Thesen zu Forschungsbedingungen der Erziehungswissenschaft im Themenfeld Inklusion. Eine Lektüre der Stellungnahme der Deutschen Gesellschaft für Erziehungswissenschaft zu Inklusion (2017)

Erziehungswissenschaft 28 (2017) 55, S. 79-88

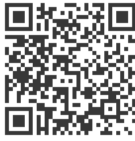

Quellenangabe/ Reference:

Puhr, Kirsten: Drei Thesen zu Forschungsbedingungen der Erziehungswissenschaft im Themenfeld Inklusion. Eine Lektüre der Stellungnahme der Deutschen Gesellschaft für Erziehungswissenschaft zu Inklusion (2017) - In: Erziehungswissenschaft 28 (2017) 55, S. $79-88$ - URN:

urn:nbn:de:0111-pedocs-152220 - DOI: 10.25656/01:15222

https://nbn-resolving.org/urn:nbn:de:0111-pedocs-152220

https://doi.org/10.25656/01:15222

in Kooperation mit / in cooperation with:

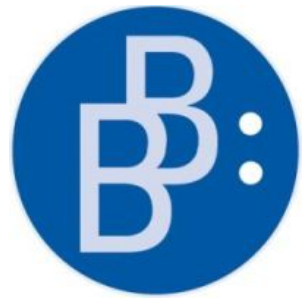

https://www.budrich.de

\section{Nutzungsbedingungen}

Dieses Dokument steht unter folgender Creative Commons-Lizenz: $\mathrm{http}: / /$ creativecommons.org/licenses/by-nd/4.0/deed.de - Sie dürfen das Werk bzw. den Inhalt vervielfältigen, verbreiten und öffentlich zugänglich machen, solange Sie den Namen des Autors/Rechteinhabers in der von inm festgelegten Weise nennen und das Werk bzw. diesen Inhalt nicht bearbeiten, abwandeln oder in anderer Weise verändern.

Mit der Verwendung dieses Dokuments erkennen Sie die

Nutzungsbedingungen an.

\section{Terms of use}

This document is published under following Creative Commons-License: http://creativecommons.org/licenses/by-nd/4.0/deed.en - You may copy, distribute and transmit, adapt or exhibit the work in the public as long as you attribute the work in the manner specified by the author or licensor. You are not allowed to alter or transform this work or its contents at all.

By using this particular document, you accept the above-stated conditions of use.

\title{
(c) $(1)$
}

\section{Kontakt / Contact:}

\section{peDOCS}

DIPF | Leibniz-Institut für Bildungsforschung und Bildungsinformation Informationszentrum (IZ) Bildung

E-Mail: pedocs@dipf.de

Internet: www.pedocs.de

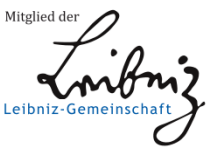




\section{Erziehungswissenschaft}

Mitteilungen der Deutschen Gesellschaft für Erziehungswissenschaft (DGfE)

Heft 55

28. Jahrgang 2017

ISSN 0938-5363

Verlag Barbara Budrich 


\section{INHALTSVERZEICHNIS}

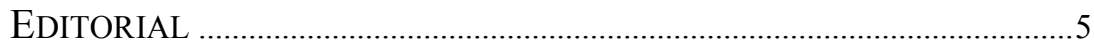

BEITRÄGE ZUM THEMA

„UNIVERSITÄT 4.0“. FOLGEN DER DIGITALISIERUNG

AKADEMISCHER LEHRE UND FORSCHUNG

Christian Swertz

Orientierungskönnen in der Leonardo-Welt

Manuela Pietraß

Was ist das Neue an „digitaler Bildung“? Zum hochschuldidaktischen

Potenzial der elektronischen Medien

Richard Stang

Lernraumgestaltung an Universitäten. Zur Relevanz physischer

Lernräume im Kontext der Digitalisierung...

Katharina Walgenbach

Elitebildung für alle? Massive Open Online Courses (MOOCs)

Ulf-Daniel Ehlers

Hochschulbildung digital. Abschied vom Ideal der Universitas?

Timo Hoyer \& Fabian Mundt

Personalisiertes Studieren, reflektiertes Lernen. Eine Analyse des

Studierverhaltens in digital gestützter Lehre.....

Ines Langemeyer \& Sabrina Schmid

Forschendes Lernen durch Mediengestaltung. Analysen zum

expansiven Lernen.

AllgEMEINE BEITRÄGE

Kirsten Puhr

Drei Thesen zu Forschungsbedingungen der Erziehungswissenschaft im Themenfeld Inklusion.

Eine Lektüre der Stellungnahme der Deutschen Gesellschaft für

Erziehungswissenschaft zu Inklusion (2017) .79 


\section{MitTEILUNGEN DES VORSTANDS}

Stellungnahme der Deutschen Gesellschaft für Erziehungswissenschaft (DGfE) zur Archivierung, Bereitstellung und Nachnutzung qualitativer Forschungsdaten in der Erziehungswissenschaft

Stellungnahme des DGfE-Vorstands zu den Reaktionen auf den

Beschluss, Hartmut von Hentig den Ernst-Christian-Trapp-Preis

abzuerkennen

\section{BERICHTE AUS DEN SEKTIONEN}

Sektion 1 - Historische Bildungsforschung......

Sektion 2 - Allgemeine Erziehungswissenschaft

Sektion 3 - Interkulturelle und International Vergleichende Erziehungswissenschaft (SIIVE)

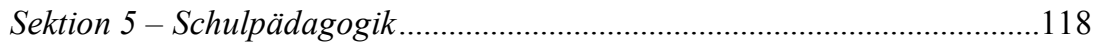

Sektion 8 - Sozialpädagogik und Pädagogik der frühen Kindheit................122

Sektion 9 - Erwachsenenbildung ............................................................130

Sektion 11 - Frauen- und Geschlechterforschung .......................................132

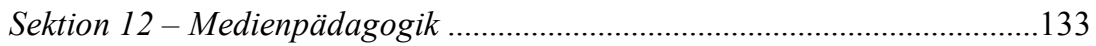

NOTIZEN

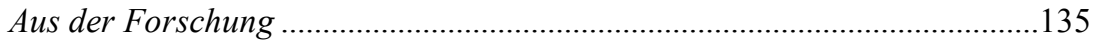

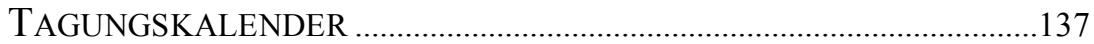

PERSONALIA

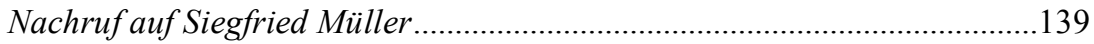




\section{AllgemeINe BeITRÄGE}

\section{Drei Thesen zu Forschungsbedingungen der Erziehungswissenschaft im Themenfeld Inklusion}

Eine Lektüre der Stellungnahme der Deutschen Gesellschaft für Erziehungswissenschaft zu Inklusion (2017)

\section{Kirsten Puhr}

Auch wenn Inklusion „kein neues Thema in der Erziehungswissenschaft“ (Hascher/Kessl 2015, S. 5) ist, erfreut es sich zunehmender Beachtung in der Erziehungswissenschaft. Das mag der ,verstärkte[n] öffentliche[n] wie fachliche[n] Diskussion [geschuldet sein, K.P.], die wesentlich unter Verweis auf die UN-Behindertenrechtskonvention geführt wird" (DGfE 2017, S. 2).

So regt die Deutsche Gesellschaft für Erziehungswissenschaften seit einiger Zeit ,einen Verständigungs- und Positionierungsprozess zur Frage der Inklusion in erziehungswissenschaftlicher Perspektive" (Hascher/Kessl 2015, S. 5) an. Als aktueller Beitrag in diesem Prozess stellt sich die Stellungnahme der Deutschen Gesellschaft für Erziehungswissenschaft zum Thema Inklusion: Bedeutung und Aufgabe der Erziehungswissenschaft (DGfE 2017) zur Diskussion. Die Stellungnahme verortet die Erziehungswissenschaft unter anderem mit der Aufgabe, ,,aus wissenschaftlicher Perspektive Stellung zu den bildungspolitischen, konzeptionellen und praktischen Fragen zu beziehen, die sich im Kontext von Inklusion stellen" (ebd., S. 2). Das Vorwort markiert zum einen ,die begriffliche Klärung und Vergewisserung darüber, was in welchem Kontext jeweils unter Inklusion zu verstehen ist und welche normativen Vorstellungen dem jeweiligen Verständnis zugrunde liegen“" (ebd.) als notwendig. Zum anderen wird eine ,bildungs- und sozialpolitische Verständigung“ aufgerufen, für die es erforderlich sei, „die strukturellen Rahmenbedingungen und den gesellschaftlichen Kontext der Bemühungen um Inklusion in die Reflexion einzubeziehen" (ebd.). In dem Zusammenhang wird die Erziehungswissenschaft ausdrücklich als Disziplin mit Expertise im „Themenfeld Inklusion“ (ebd., S. 6) vorgestellt.

Dieses (Selbst)Zeugnis legitimiert sich im Verweis auf ,umfangreiche Auseinandersetzungen mit Fragen von Behinderung und Benachteiligung, sozialer Ungleichheit, Diversity und Heterogenität aus den sonder- und integrationsbzw. inklusionspädagogischen Diskussionen [...], aber auch auf Beiträge aus der Allgemeinen Erziehungswissenschaft, der Frauen- und Geschlechterfor- 
schung, der Interkulturellen Pädagogik, der empirischen Bildungswissenschaft und vielen anderen Teildisziplinen" (DGfE 2017, S. 2). Die Stellungnahme markiert, mit der Bewertung einer „Vielfalt und Vielfältigkeit erziehungswissenschaftlicher Wissensbestände und Forschungszugänge" diese Referenzen als Ressource, ,auf die die zukünftige Forschung und Lehre zum Themenfeld Inklusion zurückgreifen kann“ (ebd., S. 6). Zudem wird die Erziehungswissenschaft unter dem Stichwort „Entwicklung erziehungswissenschaftlicher Wissensbestände“ als Herausgeforderte vorgestellt; mit der Prämisse „eigene ,blinde Flecken' zu erkennen und sich in Bezug auf ihre Forschungs- und Theorieperspektiven veränderungsbereit zu zeigen“" (ebd.).

Die hier vorgestellte Lektüre dieser Stellungnahme greift diese Anregungen auf. Sie richtet ihre Aufmerksamkeit auf den Anspruch der „Reflexion der eigenen Forschungsbedingungen“ (ebd., S. 8) der Erziehungswissenschaft im „Themenfeld Inklusion“ (ebd., S. 6). Irritiert durch den singulären exemplarischen Verweis auf Forschungsgelder ,(z.B. Finanzierung)“ (ebd., S. 8) als zu reflektierende Forschungsbedingungen stelle ich drei Thesen zur Diskussion, welche drei der vielfältigen weiteren Forschungsbedingungen aufrufen, die ich im Text der Stellungnahme lese.

So verstehe ich ,ein sozialwissenschaftliches Verständnis von Lernen und Bildung sowie von Behinderung und Partizipation“ (ebd., S. 4f.) als eine Forschungsbedingung, weil sich im Text der Stellungnahme mit ihm der Anspruch der Anknüpfung von erziehungswissenschaftlichen Überlegungen und Analysen (ebd., S. 4) im Themenfeld Inklusion verbindet. Grundlage meiner ersten These ist die Beobachtung, dass die Berufung auf ein sozialwissenschaftliches Verständnis von Partizipation als ambivalente Voraussetzung erziehungswissenschaftlicher Forschungen mit blinden Flecken verstanden werden kann.

Als eine weitere Forschungsbedingung im Themenfeld Inklusion lese ich differente Konzepte von Behinderungen und Benachteiligungen. Meine zweite These liest blinde Flecken in der Ausrichtung erziehungswissenschaftlicher Forschungen an ein interaktionistisches Verständnis von Behinderung der Behindertenrechtskonvention der Vereinten Nationen (vgl. ebd., S. 4), welches die Stellungnahme vorschlägt. Die Problematik dieser Ausrichtung lässt sich exemplarisch an einer erziehungswissenschaftlichen Reflexion sozialpolitischer Inklusionsbemühungen zeigen. Der Text „Das neue SGB VIII Fragen aus der Perspektive der Lebensweltorientierung“ (Thiersch 2016) markiert die Berücksichtigung aller „Heranwachsenden und Kinder im Kontext der neueren Inklusionsdebatte [...] also nach einer Integration der jungen Menschen mit Behinderungen"einerseits als „lange angemahnte Forderung" (ebd., S. 283). Andererseits beobachtet er, dass diese Debatte nicht im Sinne „einer Inklusion aller [...], die in beeinträchtigten, benachteiligten Verhältnissen leben, also in den Unterdrückungsstrukturen von Armut, fehlenden Ressourcen und dadurch bedingter Exklusion oder von Geschlecht und ethnischer Zugehörigkeit“" (ebd.) geführt wird. 
Meine dritte These wendet sich der Einschätzung zu, der Inklusionsanspruch „stellt für die Disziplin der Erziehungswissenschaft ein Diskussionsangebot dar, sich mit der (impliziten) Normativität erziehungs- und bildungswissenschaftlicher sowie pädagogischer Konzepte, Theorien und Modelle auseinanderzusetzen und Behinderungen und Benachteiligungen, die in pädagogischen Organisationen respektive Interaktionen hervorgebracht werden, zu beschreiben und zu reflektieren" (DGfE 2017, S. 4). Dieser Einsatz ist der Frage geschuldet: Was können Formen (erziehungs)wissenschaftlicher Reflexion (Beobachtungen zweiter Ordnung) anders beschreiben und analysieren als Beobachtungen erster Ordnung? In diesem Zusammenhang lese ich Widersprüche als Forschungsbedingungen von Erziehungswissenschaft und frage nach Potenzialen des Anspruchs ,der Überwindung respektive der Reflexion von Widersprüchen“"(ebd.).

\section{Kritik des Anspruchs der Anknüpfung erziehungswissenschaftlicher Forschungen im Themenfeld Inklusion an ein sozialwissenschaftliches Verständnis von Lernen und Bildung sowie von Behinderung und Partizipation}

These 1: Der Anspruch der Anknüpfung an ein sozialwissenschaftliches Verständnis von Lernen und Bildung sowie von Behinderung und Partizipation (vgl. DGfE 2017, S. 4f.) erscheint einschränkend und blind für widerstreitende wissenschaftliche Positionierungen im Themenfeld Inklusion/Exklusion.

Das Themenfeld Inklusion/Exklusion zeichnet sich durch differente sozialwissenschaftliche und weitere (z. B. bildungs- und politikphilosophische) Positionierungen aus. Derartig vielfältige Referenzen legitimieren widerstreitende Einsätze erziehungswissenschaftlicher Forschungen (auch im Themenfeld Inklusion) und stärken die „Vielfalt und Vielfältigkeit erziehungswissenschaftlicher Wissensbestände und Forschungszugänge“" (ebd., S. 6).

Dafür ein Beispiel von vielen Möglichen: Die Stellungnahme der DGfE benennt explizit „Inklusion als Forschungs- und Diskussionsauftrag an die Erziehungswissenschaft" (ebd., S. 7). Meine Lektüre vermisst in dieser Positionierung das Konzept der Exklusion. Die Konstruktion dieses blinden Flecks ist einem soziologischen Zugang geschuldet, welcher (in der Bezeichnung von Inklusion) Exklusion als die unbezeichnete Seite der Differenz Inklusion/Exklusion beobachtet. Die Frage nach der Differenz Inklusion/Exklusion drängt sich der Lektüre insofern auf, als dass die Stellungnahme implizit auf Exklusion als Forschungs- und Diskussionsanlass zu verweisen scheint, etwa im Aufrufen eines Bedarfs an empirischen Analysen ,jener Strukturen und Barrieren, die ihre Umsetzung [die der Inklusion, K.P.] aktuell einschränken oder verhindern" (ebd.). Eine Vorstellung von Exklusion verbindet 
sich nach meiner Lesart auch mit der Formulierung eines Gebotes mehrdimensionaler/intersektionaler Ausrichtung erziehungswissenschaftlicher Betrachtungen von ,z. B. Benachteiligungspraktiken oder Behinderungsdynamiken“ (DGfE 2017, S. 7) im „Zusammenspiel“ (ebd., S. 8) von Differenz- und Ungleichheitsdimensionen und nicht zuletzt im Verweis auf ,die sozialwissenschaftlichen Diskurse der Erziehungswissenschaft" (ebd., S. 4).

Mit der Frage nach Vorstellungen von Exklusion in den Sozialwissenschaften lese ich in der "Rhetorik der Exklusion“ (Farzin 2011). Hier werde ich von einer "steilen Begriffskarriere in der Sozialwissenschaft" (ebd., S. 18) informiert, die der „Vokabel [...] der sozialen Exklusion“ (ebd., S. 11) zugeschrieben wird. Zu lesen ist von einem ,, Minimalkonsens“ unterschiedlicher Definitionsansätze“, nach dem Exklusion „einen integrierten und umfassenden Ausschluss einzelner Personen oder Personengruppen aus verschiedenen gesellschaftlichen Kontexten" (ebd.) bezeichnet. Insofern scheint es nahezuliegen, Inklusion, im Sinne ,universale[r] Partizipationsmöglichkeiten aller Gesellschaftsmitglieder in allen Teilhabebereichen" $z u$ fassen und Exklusion als Gegenbegriff, in der Bedeutung der ,Abwesenheit von gesellschaftlichen Teilhabemöglichkeiten" (ebd., S. 19). Sozialwissenschaftliche Kritiken eines solchen Verständnisses von Inklusion und Exklusion als Gegenbegriffe fordern jedoch ein differenztheoretisches Konzept von Inklusion/ Exklusion, das Fragen danach ermöglicht, ,welche Formen sozialer Partizipation als Exklusion und somit als gesamtgesellschaftliches Problem bestimmt werden und wie auf diese Weise zugleich Kriterien für ,normale' Partizipationsformen, also Inklusion, implizit definiert werden“" (ebd., S. 20).

Derartig ambivalente (sozial)wissenschaftliche Positionierungen im Themenfeld Inklusion/Exklusion können als produktive Referenzen/Forschungsbedingungen erziehungswissenschaftlicher Forschungen verstanden werden, vor allem weil die Stellungnahme explizit den Auftrag formuliert, „den Blick auf strukturelle Exklusions- bzw. Marginalisierungsbedingungen zu richten, die sich z.B. in Diskriminierungs- und Etikettierungsprozessen zeigen“" (DGfE 2017, S. 5). Eine solche Aufmerksamkeit kann erziehungswissenschaftliche Einsätze auch auf weitere wissenschaftliche Referenzen stoßen, z. B. politikphilosophische, die Konzepte von Inklusion/Exklusion mit Fragen „einer radikalen Heterogenität des Sozialen und des Subjekts [...] als [kaum] programmatisch lösbares Problem“" (Mayer 2014, S. 210) diskutieren. Die Erziehungswissenschaft kann zudem auf zahlreiche Expertisen sowohl in den sogenannten Differenz-Pädagogiken als auch in den Allgemeinen Erziehungswissenschaften zurückgreifen, Beiträge in denen ,die Frage nach Inklusion zentral [erscheint, K.P.] auch wenn sie [...] nicht unter diesem Titel“" (Hascher/Kessl 2015, S. 5) diskutiert wird. Ich verweise exemplarisch auf das Konzept der Destabilisierung mit dem ,Anspruch auf gesellschaftspolitische Gestaltungsmacht“ (Engel 2002, S.9) im Text „Wider die Eindeutigkeit. Sexualität und Geschlecht im Fokus queerer Politik der Repräsentationen“ 
(Engel 2002) und die kritische Diskussion des Begriffs Partizipation ,als ein Versprechen“ (Ahrens/Wimmer 2014, S. 175f.) im Aufsatz „Das Demokratieversprechen des Partizipationsdiskurses. Die Gleichsetzung von Demokratie und Partizipation“" (Ahrens/Wimmer 2014). Mit derartigen Einsätzen können sich, unter Berufung auf differente wissenschaftliche Referenzen im Themenfeld Inklusion/Exklusion, verschiedenartige Möglichkeiten öffnen, erziehungswissenschaftliche Forschungspositionen im Themenfeld Inklusion/Exklusion zu stärken, die explizit als widerständige Einsätze und nicht (nur) als „aber auch [...] Beiträge“ (DGfE 2017, S. 2) und insofern als Ergänzungen der ,sonder- und integrations- bzw. inklusionspädagogischen Diskussionen der letzten Jahre“" (ebd.) zu verstehen sind.

Kritik der normativen Ausrichtungen erziehungswissenschaftlicher Positionierungen im Themenfeld Inklusion/Exklusion an der Behindertenrechtkonvention der Vereinten Nationen als Bekräftigung der Allgemeinen Menschenrechte (vgl. DGfE 2017, S. 3)

These 2: Normative Ausrichtungen erziehungswissenschaftlicher Positionierungen im Themenfeld Inklusion an der Behindertenrechtskonvention der Vereinten Nationen (vgl. DGfE 2017, S. 3) fokussieren ihre Aufmerksamkeit vordergründig auf Behinderung, im Sinne personifizierender Zuschreibungen, und verschenken damit einen wesentlichen Teil ihres Potenzials für kritische Gesellschaftsanalysen.

Die Annahme eines Zusammenspiels von Differenz- und Ungleichheitsdimensionen in Benachteiligungspraktiken oder Behinderungsdynamiken (vgl. DGfE 2017, S. 5) kann sich mit vielfältigen erziehungswissenschaftlichen Einsätzen verbinden, z.B. mit einer ,menschenrechtlichen Perspektive universeller Anerkennung von Diversität, die in der Gewährleistung von Rechtsgleichheit und Nicht-Diskriminierung materialisiert werden soll“" (ebd., S. 2), oder mit differenztheoretisch begründeten Vorstellungen der Wechselseitigkeit von Inklusions- und Exklusionspraktiken und der Unterscheidung von Teilhabe und Teilnahme oder mit grundsätzlichen Kritiken an eindimensionalen Kategorisierungen und am Anspruch der Inklusion in bestehende soziale Strukturen. Angesichts dieser Vielstimmigkeit kann die Stellungnahme das Thema Inklusion ,als Diskursangebot für die Bündelung von Fragen der Bildungsgerechtigkeit sowie der Partizipation" (ebd., S. 3), des erziehungswissenschaftlichen Interesses an der „Ermöglichung gleichberechtigter Partizipation und sozialer Zugehörigkeit für unterschiedliche Akteur/-innen mit differenten Interessen und Bedürfnissen“ sowie an ,sozialen, also interaktiv hergestellten, Formen von Behinderung und Benachteiligung [...] mit Fokus auf 
Bildungs- und Erziehungsverhältnisse“ (DGfE 2017, S. 4) verorten. Mit diesen Verankerungen lassen sich erziehungswissenschaftliche Inklusions/Exklusions-Forschungen als vielgestaltige Perspektiven kritischer Gesellschaftsanalysen verstehen, die sich für diverse politische, ökonomische und kulturelle Aspekte des Inklusionsdiskurses öffnen können und dabei „die strukturellen Rahmenbedingungen und den gesellschaftlichen Kontext der Bemühungen um Inklusion“ (ebd., S. 2) einbeziehen.

In diesem Diskurs ermöglicht die Artikulation einer ,,allgemeine[n] Gleichheit aller Menschen“ (Mouffe 2010, S. 76) mit „Bezug auf die ,Menschheit“ und den polemischen Einsatz der ,Menschenrechte' die permanente Infragestellung der Exklusionsformen“" (ebd., S. 82) politischer Praxen, wie z.B. die „einer demokratischen Gesellschaftsentwicklung“ (DGfE 2017, S. 4). Die Öffnung für einen politischen Aspekt des Inklusionsdiskurses kann mit dieser Möglichkeit kritischer Gesellschaftsanalysen zugleich die Differenz Inklusion/Exklusion [als, K.P.] „Das Paradox der Demokratie“ (Mouffe 2010, S. 75ff.) lesen. Die Stellungnahme der DGfE ruft die Behindertenrechtskonvention der Vereinten Nationen als Bekräftigung der Allgemeinen Menschenrechte auf (vgl. DGfE 2017, S. 3) und legitimiert in ihrem Text normative Ausrichtungen erziehungswissenschaftlicher Positionierungen im Themenfeld Inklusion ausschließlich auf diese. Die Berufung auf die UN-BRK findet sich in allen Ausführungen, die Forschungsbedingungen im Sinne von „Ausgangssituation und Kontext" (ebd.) des Themas Inklusion aus erziehungswissenschaftlicher Sicht thematisieren: ,(a) als bildungs- und sozialpolitischer Impuls; (b) als ethischer Orientierungshorizont [...]; und (c) als Diskursangebot für die Bündelung von Fragen der Bildungsgerechtigkeit sowie der Partizipation" (ebd., Hervorhebungen im Original). Diese Referenz legitimiert sich mit einem ,interaktionistischen Verständnis von Behinderung“, das ,einen Blick auf die sozialen, also interaktiv hergestellten, Formen von Behinderung und Benachteiligung" (ebd., S. 4) öffnet. Als Argument für diese Lesart kann angeführt werden, dass ein „Kerngedanke dieses Modells lautet, dass Behinderung kein individualisierter Zustand ist, sondern eine kontextbedingte Lebens- und Handlungssituation“" (Dederich 2016, S. 108).

Als Gegenargument ließe sich einwenden, dass als Ausgangspunkt für solcher Art Begriffsbestimmungen von Behinderungen diagnostizierbare Beeinträchtigungen von Personen/Personengruppen im Sinne personifizierender „Sammelvokabel[n] für heterogene soziale Gruppen“ (Farzin 2011, S. 14) aufgerufen werden. So kann die Hervorhebung der UN-BRK als Ausgangssituation und Kontext für erziehungswissenschaftliche Positionierungen im Themenfeld Inklusion einerseits als Referenz auf ein spezifisches (unzureichend realisiertes) gesellschaftliches Inklusionsgebot gelesen werden und andererseits als Nicht-Berücksichtigung des Anspruchs an erziehungswissenschaftliche Forschungen, bei der Betrachtung von „Benachteiligungsprakti- 
ken oder Behinderungsdynamiken [...] nicht nur einzelne Differenz- und Ungleichheitsdimensionen [zu] berücksichtigen“ (DGfE 2017, S. 7f.).

Die Stellungnahme der DGfE beruft sich auf die menschenrechtliche Perspektive der UN-BRK mit einem „Potenzial zur Überwindung einer kategorialen zuschreibenden Perspektive auf Benachteiligung und Behinderung" (ebd., S. 4). Ich kann dieses Vermögen in der UN-BRK nicht finden. Ihr Potenzial lese ich in der Bekräftigung ,aller Menschenrechte und Grundfreiheiten“ (UN 2006, § 1 Satz 1; vgl. auch Präambel Absatz a-d), an denen eben auch ,alle Menschen mit Behinderungen“ (ebd., § 1 Satz 1) partizipieren können sollten. Die Förderung dieser Teilhaberechte präsentiert die $U N-B R K$ als ihren Zweck (vgl. ebd.). Dieser Zweck begründet sich als Kritik zahlreicher Beobachtungen von Einschränkungen gesellschaftlicher Teilhabemöglichkeiten, die Menschen mit Behinderungen betreffen (ebd., Präambel, Absatz e-t). In diesem Kontext ruft die Behindertenrechtskonvention der Vereinten Nationen zwar keinen ,,,individualisierenden' bzw. ,essenzialisierden' Begriff von Behinderung und Benachteiligung" (DGfE 2017, S. 5) auf, jedoch ein personifizierendes Verständnis von Behinderung. So ist in $\S 1$, Satz 2 zu lesen:

„Zu den Menschen mit Behinderungen zählen Menschen, die langfristige körperliche, seelische, geistige oder Sinnesbeeinträchtigungen haben, die in Wechselwirkung mit verschiedenen Barrieren ihre volle und wirksame Teilhabe gleichberechtigt mit anderen an der Gesellschaft behindern können.“(UN 2006, § 1, Satz 2)

Dem gegenüber thematisiert die Stellungnahme der DGfE ausdrücklich die Überwindung personifizierender Zuschreibungen von Behinderungen und Benachteiligungen (vgl. DGfE 2017, S. 5) als konkretisierten Auftrag der Erziehungswissenschaft, als einen Aspekt ihres Beitrages ,zur Reflexion aktueller Umsetzungen sowie bestehender struktureller und interaktiv hergestellter Partizipationseinschränkungen“" (ebd.). Ich stelle in Frage, dass das Behinderungskonzept der $U N-B R K$ dazu in aufgerufener Weise geeignet ist und gebe zu bedenken, dass erziehungswissenschaftliche Inklusions/ExklusionsForschungen mit Blick auf diverse Behinderungs- und Benachteiligungsdimensionen sowie unter Berücksichtigung verschiedener Differenz- und Ungleichheitstheorien über ein weitaus vielseitigeres Potenzial verfügen.

\section{$\mathrm{Zu}$ möglichen Bedeutungen von Widersprüchen als Forschungsbedingungen von Erziehungswissenschaft}

These 3: Die Frage nach Positionierungen im Themenfeld Inklusion/Exklusion kann in dem Anspruch ,,der Überwindung respektive der Reflexion von Widersprüchen" (ebd., S. 4) differente Antworten finden, die grundlegend das Selbstverständnis der Erziehungswissenschaft als Wissenschaft betreffen. 
Mit dem Anspruch „,der Überwindung respektive der Reflexion von Widersprüchen“ in Auseinandersetzungen „mit der (impliziten) Normativität erziehungs- und bildungswissenschaftlicher sowie pädagogischer Konzepte, Theorien und Modelle“ sowie in Beschreibungen und Reflexionen von „Behinderungen und Benachteiligungen, die in pädagogischen Organisationen respektive Interaktionen hervorgebracht werden" (DGfE 2017, S. 4) positioniert die Stellungnahme der DGfE nach meiner Lesart Einsätze der Erziehungswissenschaft als differente Perspektiven kritischer Gesellschaftsanalysen.

Meine Frage nach möglichen Bedeutungen von Widersprüchen als Forschungsbedingungen nimmt ihren Ausgangspunkt in der Frage, ,wie die Welt in der Welt beobachtet werden könne, also wie die Welt sich selbst beobachte" (Luhmann 1997, S. 1114). Für die Frage nach Weltbeschreibungen in Weisen kritischer Gesellschaftsanalysen kann die „Form der Selbstbeschreibung“ (Beobachtung erster Ordnung) von der „Form der Reflexion“ (ebd., S. 1115) (Beobachtung zweiter Ordnung) unterschieden werden. Bezogen auf das Themenfeld Inklusion/Exklusion wäre mit einer solchen Unterscheidung davon auszugehen, dass Selbstbeschreibungen einer Gesellschaft (Beobachtungen erster Ordnung) ein grundlegendes Verständnis von Inklusion/Exklusion benötigen, das „,sich dieser Aufgabe anpassen muß“ (ebd.). So wird aus einer soziologisch-systemtheoretischen Perspektive für eine funktional differenzierte Gesellschaft ein grundlegendes Inklusionsgebot und ein ebensolches Exklusionsverbot angenommen, obwohl ungleiche Chancen beschrieben und kritisch analysiert werden, weil diese als funktionslos reproduzierte Ungleichheiten zum Problem werden. Aus einer politikphilosophischen Perspektive lässt sich erklären, dass das Selbstverständnis einer liberaldemokratischen Gesellschaft gleiche Inklusionsmöglichkeiten für alle proklamiert, die sich auf eine ,allgemeine Gleichheit aller Menschen, [als eine] nichtpolitische Form der Gleichheit" (Mouffe 2010, S. 76) beruft, und zugleich Sozialgesetzänderungen als inklusionsorientierte legitimiert, „die unverhohlen auf eingeschränkte Leistungen für unbeteiligte minderjährige Flüchtlinge" (Peters 2016, S. 280) zu zielen scheinen. Dieses Zugleich als legitime Selbstbeschreibung einer liberal-demokratischen Gesellschaft bezogen auf das Themenfeld Inklusion/Exklusion kann sich mit der Vorstellung begründen, dass die „Identität einer demokratischen politischen Gemeinschaft auf der Möglichkeit einer ,Grenzziehung ' [...] basiert, [...], dass die Demokratie stets Inklusions-/Exklusionsverhältnisse umfaßt" (Mouffe 2010, S. 81).

Formen erziehungswissenschaftlicher Reflexionen (Beobachtungen zweiter Ordnung) können sozial- und bildungspolitische Positionierungen wie die zitierte kritisieren, weil diese mit ihren Grenzziehungen/impliziten Exklusionen ungleiche Teilhabechancen konstituieren, mit denen die liberal-demokratische Gesellschaft in ,Widerspruch zu ihrer universalistischen Rhetorik gerät“ (ebd.). Solche kritischen Beobachtungen können sich z. B. mit Reflexionen von Widersprüchen als Formen analytischer Kritik erziehungswissen- 
schaftlicher Inklusions/Exklusions-Forschungen an der Sozialpolitik der Gesellschaft und/oder als grundsätzliche menschenrechtlich legitimierte Kritik am uneingelösten Anspruch, ,gleichberechtigter Partizipation und sozialer Zugehörigkeit" (DGfE 2017, S. 4) zeigen. Derartige Kritiken können sich als „konkurrierende Gesellschaftsbeschreibung[en]“ (Luhmann 1997, S. 1116) eines Beobachters erster Ordnung inszenieren. Ein solches erziehungswissenschaftliches Wissen kann sich z. B. mit visionären Vorstellungen eines „,nicht-diskriminierenden und auf Chancengleichheit ausgerichteten Bildungssystems“ (DGfE 2017, S. 3) oder auch mit „weitgefächterte[n] Expertisen“ (ebd., S. 2) legitimieren. Derartige Kritiken können jedoch auch, z. B. wenn sie mit dem Anspruch der „Überwindung“ (ebd., S. 4) von Widersprüchen auftreten, als „Attitüden des Besserwissens“ (Luhmann 1997, S. 1115) beobachtet werden, die „bildungs- und sozialpolitische Verständigung“ (DGfE 2017, S. 2) selten befördern.

Ein konstitutives Verständnis von Widersprüchen als Forschungsbedingungen der Erziehungswissenschaft kann davon ausgehen, dass Formen wissenschaftlicher Reflexion (als Beobachtungen zweiter Ordnung) anderes sehen können als die beobachteten menschenrechtsbasierten und/oder bildungs- und sozialpolitischen Selbstbeschreibungen. Das kritische Potenzial erziehungswissenschaftlicher Inklusions/Exklusionsforschungen bezöge sich „auf den blinden Fleck des beobachteten Beobachters, auf das, was er nicht sehen kann. Und das, was in der Gesellschaft als natürlich und notwendig gilt, wird in dieser Perspektive etwas Artifizielles und Kontingentes" (ebd., S. 1119). Bezogen auf die zur Diskussion gestellte These wäre in dieser Lesart die Reflexion von Widersprüchen als ein anderer Fall/eine andere Aussage zu verstehen als die Überwindung von Widersprüchen, weil aus einer Reflexion/Beobachtung zweiter Ordnung nicht folgt, „daß man auch sagen könnte, wie es anders zu machen wäre" (ebd.). Mit einem solchen Zugang können sich Reflexionen der eigenen Forschungsbedingungen ergeben, die sich neben den beispielhaft genannten Finanzierungen (DGfE 2017, S. 8), den ,strukturellen Rahmenbedingungen und de[m] gesellschaftlichen Kontext der Bemühungen um Inklusion“ (ebd., S. 2) auch auf erziehungswissenschaftliche Selbstverständnisse im Themenfeld Inklusion/Exklusion beziehen. Dafür bietet die Stellungnahme der Deutschen Gesellschaft für Erziehungswissenschaft zum Thema Inklusion: Bedeutung und Aufgabe der Erziehungswissenschaft (DGfE 2017) vielfache Anlässe.

Kirsten Puhr, Prof. Dr., ist Hochschullehrerin am Institut für Sonderpädagogik an der Pädagogischen Hochschule Heidelberg. 


\section{Literatur}

Ahrens, Sönke/Wimmer, Michael (2014): Das Demokratieversprechen des Partizipationsdiskurses. Die Gleichsetzung von Demokratie und Partizipation. In: Schäfer, A. (Hrsg.): Hegemonie und autorisierende Verführung. Paderborn: Schöningh, S. 175-199.

Deutsche Gesellschaft für Erziehungswissenschaft (DGfE) (2017): Inklusion: Bedeutung und Aufgabe der Erziehungswissenschaft. Stellungnahme der DGfE. www.dgfe.de/fileadmin/OrdnerRedakteure/Stellungnahmen/2017 Inklusion_Stellungsnahme.pdf [Zugriff: 06. Februar 2017].

Engē, Antke (2002). Wider die Eindeutigkeit. Sexualität und Geschlecht im Fokus queerer Politik der Repräsentationen. Frankfurt am Main: Campus.

Dederich, Markus (2016): Behinderung. In: Dederich, M./Beck, I./Bleidick U./Antor, G. (Hrsg.): Handlexikon der Behindertenpädagogik. Schlüsselbegriffe aus Theorie und Praxis. 3., erweiterte und überarbeitete Auflage. Stuttgart: Kohlhammer, S. 107-110.

Farzin, Sina (2011): Die Rhetorik der Exklusion. Zum Zusammenhang von Exklusionssemantik und Sozialtheorie. Weilerswist: Velbrück Wissenschaft.

Hascher, Tina/Kessl, Fabian (2015): Inklusion eine erziehungswissenschaftliche Perspektive. Editional. In: Erziehungswissenschaft. Mitteilungen der Deutschen Gesellschaft für Erziehungswissenschaft 51, 26, S. 5-6.

Luhmann, Niklas (1997): Die Gesellschaft der Gesellschaft. Frankfurt am Main: Suhrkamp.

Mayer, Ralf (2014): Produktivität von Heterogenität. In: Koller, H.-C./Casale, R./Ricken, N. (Hrsg.): Heterogenität - Zur Konjunktur eines pädagogischen Konzepts. Paderborn: Schöningh, S. 201-218.

Mouffe, Chantal (2010): Inklusion/Exklusion: Das Paradox der Demokratie. In: Weibel, P./Žižek, S. (Hrsg.): Inklusion: Exklusion. Probleme des Postkolonialismus und der globalen Migration. 2. Auflage, Wien: Passagen, S. 75-90.

Peters, Friedhelm (2016): SGB VIII-Novilierung die Ambivalenzen diskutieren, Optionen erhalten! In: Forum Erziehungshilfen 22, 5, S. 280-282.

Thiersch, Hans (2016): Das neue SGB VIII Fragen aus der Perspektive der Lebensweltorientierung. In: Forum Erziehungshilfen 22, 5, S. 283-287.

Vereinte Nationen (UN) (2006): Übereinkommen über die Rechte von Menschen mit Behinderungen. In: Netzwerk Menschenrechte (Hrsg.): Behindertenrechtkonvention. Schattenübersetzung. Korrigierte Fassung der zwischen Deutschland, Liechtenstein, Österreich und der Schweiz abgestimmten Übersetzung. www.behindertenrechtskonvention.info/inhalte [Zugriff: 12. Januar 2017]. 